\title{
BOOLEAN ALGEBRAS WITH NO RIGID OR HOMOGENEOUS FACTORS
}

BY

\author{
PETR ŠTĚPÁNEK
}

\begin{abstract}
A simple construction of Boolean algebras with no rigid or homogeneous factors is described. It is shown that for every uncountable cardinal $\kappa$ there are $2^{\kappa}$ isomorphism types of Boolean algebras of power $\kappa$ with no rigid or homogeneous factors. A similar result is obtained for complete Boolean algebras for certain regular cardinals. It is shown that every Boolean algebra can be completely embedded in a complete Boolean algebra with no rigid or homogeneous factors in such a way that the automorphism group of the smaller algebra is a subgroup of the automorphism group of the larger algebra. It turns out that the cardinalities of antichains in both algebras are the same. It is also shown that every $\kappa$-distributive complete Boolean algebra can be completely embedded in a $\kappa$-distributive complete Boolean algebra with no rigid or homogeneous factors.
\end{abstract}

Introduction. For every nonzero element $u$ of a Boolean algebra $B$, the principal ideal consisting of all elements $v, v \leqslant u$, with the restricted operations is a Boolean algebra. We shall call it a factor of $B$ and denote it by $B \uparrow u$. If $B \uparrow u$ is a homogeneous (rigid) Boolean algebra, we shall call it a homogeneous (rigid) factor of $B$. Clearly, every factor of a complete Boolean algebra is a complete algebra, too. Every complete Boolean algebra $B$ can be represented in a unique way as the product of three factors, namely $B=H \times R \times D$, where $R$ is the product of a family of rigid factors of $B, H$ is the product of homogeneous factors of $B$ and $D$ is a complete Boolean algebra with no rigid or homogeneous factors.

Studying the automorphism groups of Boolean algebras, McKenzie and Monk [8] showed that the problem reduces to characterization of the automorphism groups of the three types of Boolean algebras corresponding to the above decomposition. They proved representation theorems for the products of rigid Boolean algebras and for the products of homogeneous Boolean algebras. The characterization of automorphism groups of Boolean algebras with no rigid or homogeneous factors remained an open problem. At that time, no example of such algebras was known. The existence of Boolean algebras with no rigid or homogeneous factors was proved by Balcar and the author in [14]. The aim of this paper is to describe a simpler construction of such algebras and to prove new results about them. The paper is organized as follows. §1 describes the basic construction which was motivated by Shelah's construction of

Received by the editors September 20, 1980.

1980 Mathematics Subject Classification. Primary 06E05, 06E10.

Key words and phrases. Boolean algebras with no rigid or homogeneous factors, automorphisms, embeddings and isomorphism types of BA's, chain conditions, distributivity. 
rigid Boolean algebras [12]. Isomorphism types of Boolean algebras with no rigid or homogeneous factors are studied in §2. Using some ideas of Monk and Rassbach [9], we shall show that for every uncountable cardinal $\kappa$, there are $2^{\kappa}$ isomorphism types of (noncomplete) Boolean algebras of power $\kappa$ with no rigid or homogeneous factors. If $\kappa$ is regular and $\kappa^{\aleph_{0}}=\kappa$, we have the same result for complete Boolean algebras. In $\S \S 3$ and 4, we prove two embedding theorems. We shall show that every Boolean algebra $B$ can be completely embedded in a complete Boolean algebra $C$ with no rigid or homogeneous factors. The first theorem gives an embedding preserving saturatedness i.e. the cardinalities of antichains in $B$ and $C$ are the same. The second theorem gives embeddings preserving distributivity. It turns out that in both cases every automorphism of $B$ can be extended to an automorphism of $C$ and the automorphism group $\operatorname{Aut}(B)$ of $B$ is a subgroup of $\operatorname{Aut}(C)$. This result gives partial information about automorphism groups of Boolean algebras with no rigid or homogeneous factors showing that automorphism groups of these algebras reflect in some sense the structure of automorphism groups of all Boolean algebras. The above-mentioned embedding theorems are compared with embedding theorems due to Kripke [6] and Koppelberg [5] concerning embeddings into homogeneous Boolean algebras. It turns out that the saturatedness preserving embeddings impose some restrictions on automorphisms of the larger algebra and that the statement "every Boolean algebra can be completely embedded in a complete Boolean algebra with the same saturatedness and with an atomless homogeneous factor" is not a theorem of Zermelo-Fraenkel set theory (ZFC). The relationship to the results of [13] and [14] concerning embeddings into rigid Boolean algebras is also discussed and some open problems are stated.

0. Preliminaries. A Boolean algebra $B$ is a structure $\left\langle B, \wedge, \vee,-, 0_{B}, 1_{B}\right\rangle$ satisfying the usual axioms. Every Boolean algebra is partially ordered by a canonical ordering $\leqslant, 1_{B}$ being the greatest and $0_{B}$ the least element. It is well known that Boolean operations are definable from the canonical ordering and vice versa. We say that two elements $u, v$ of $B$ are disjoint if $u \wedge v=0_{B}$, otherwise we say that $u$ and $v$ are compatible. If it is clear what algebra we have in mind, we shall omit the subscripts of 0 and 1 . For every nonzero element $u$ of $B$, we shall denote by $B \uparrow u$ the principal ideal of all $v, v \leqslant u . B \uparrow u$ with the restricted operations is a Boolean algebra and we shall call it a factor of $B$. We say that $B$ is homogeneous iff for every $u, v \in B$ neither of which is equal to 0 or 1 , there is an automorphism $f$ of $B$ with $f(u)=v$. We say that $B$ is weakly homogeneous if for every two nonzero elements $u, v$ of $B$, there is an automorphism $f$ such that $f(u), v$ are compatible. It is clear that every homogeneous Boolean algebra is weakly homogeneous. We say that $B$ is a rigid Boolean algebra if the identity is the only automorphism of $B$. We say that a Boolean algebra has no rigid or homogeneous factors if every factor of it is neither rigid nor homogeneous. If $\kappa$ is a cardinal number, we say that a Boolean algebra satisfies the $\kappa$-antichain condition ( $\kappa-\mathrm{AC})$ if every antichain i.e. every set of pairwise disjoint elements of it has power less than $\kappa$. The least cardinal $\kappa$ such that $B$ satisfies the $\kappa$-antichain condition is called the saturatedness of $B$ and we denote it by $\operatorname{sat}(B)$. 
We say that $B$ is a complete Boolean algebra if the operations of join and meet can be extended to any set of elements of $B$. It is well known that every (separative) partially ordered set $(P, \leqslant)$ determines uniquely (up to isomorphism) a complete Boolean algebra $B$ such that $(P, \leqslant)$ is isomorphic to a dense subset of $B$. In particular, every Boolean algebra $B$ has a unique (up to isomorphism) completion $B^{c}$. Recall that a subset $d$ of $B$ is dense if for every nonzero element $u$ of $B$ there is a nonzero element $v$ of $d, v \leqslant u$.

It is well known that every automorphism of a Boolean algebra can be uniquely extended to an automorphism of its completion. Every automorphism of a factor $B \nmid u$ can be extended to an automorphism of $B$ e.g. if we put $f(v)=v$ for every $v$ disjoint from $u$. Consequently, if $B$ has no rigid factors then $B^{c}$ has no rigid factors, too. On the other hand, if $B$ is homogeneous, then so is $B^{c}$. To prove this, note that the saturatedness of a homogeneous Boolean algebra is the same as the saturatedness of each of its factors and use the fact that isomorphisms are preserved by products of Boolean algebras. Consequently, if $B$ has a homogeneous factor then $B^{c}$ has a homogeneous factor, too.

A set $p$ of nonzero elements of $B$ is called a partition of $B$ if every two elements of $p$ are disjoint and $\vee p=1$. We say that a partition $q$ is a refinement of $p$ if for every $v$ in $q$ there is some $u$ in $p$ such that $v \leqslant u$. Note that for every partition

$$
p=\left\{u_{i}, i \in I\right\}
$$

of a complete Boolean algebra $B$ the product $P_{i \in I}\left(B \nmid u_{i}\right)$ is a complete Boolean algebra isomorphic to $B$. On the other hand, if $B$ is the product of Boolean algebras $A_{i}, i \in I$, then there is a partition (1) such that $A_{i}$ and $B \nmid u_{i}$ are isomorphic for every $i$. We say that a dense subset $d$ is $\kappa$-closed for some cardinal $\kappa$, if for every $\alpha, \alpha \leqslant \kappa$, and every decreasing sequence $\left\langle\mu_{\xi}, \xi<\alpha\right\rangle$ of nonzero elements of $d$ there is a nonzero $u$ in $d$ such that $u \leqslant u_{\xi}$ holds for every $\xi$. We say that a complete Boolean algebra is $\kappa$-distributive if every set of $\kappa$ partitions of it has a common refinement. Note that if $B$ has a $\kappa$-closed dense subset then $B$ is $\kappa$-distributive. Let us recall the definition of the direct sum (free product) of Boolean algebras. Let $B_{1}, B_{2}$ be Boolean algebras and $\leqslant_{1}, \leqslant_{2}$ be in turn their canonical orderings. If we put $P=\left\{\left(u_{1}, u_{2}\right), u_{1}, u_{2}\right.$ are nonzero elements of $\left.B_{1}, B_{2}\right\}$ and $\left(u_{1}, u_{2}\right) \leqslant\left(v_{1}, v_{2}\right)$ iff $u_{1} \leqslant v_{1} v_{1}$ and $u_{2} \leqslant 2 v_{2}$ then $\leqslant$ is a separative partial ordering of $P$. The direct sum of $B_{1}, B_{2}$ is the complete Boolean algebra $B_{1} \oplus B_{2}$ uniquely determined by $(P, \leqslant)$. It is clear that $B_{1}$ and $B_{2}$ are completely embedded in $B_{1} \oplus B_{2}$.

The following proposition resembles the spirit of Theorem 2 of Engelking and Karlowicz [2]. We shall use it later to get bounds on the cardinalities of antichains.

Proposition. Let $\mu$ be an ordinal, $\mu \geqslant \omega$. For every $i, i \in I$, let $a(i), b(i)$ be sets of ordinals satisfying the following properties.

(2) The family a ( $i)$ is pairwise disjoint,

(3) for every two different $i, i^{\prime}$, we have $a(i) \cap b\left(i^{\prime}\right) \neq 0$ or $a\left(i^{\prime}\right) \cap b(i) \neq 0$, and

(4) every $a(i)$ is finite and the order-type of every $b(i)$ is at most $\mu$.

Then the cardinality of $I$ is at most the cardinality of $\mu$. 
Proof. Put $\Lambda=|\mu|$ and suppose that the power of $I$ is at least $\Lambda^{+}$. As it is a regular and uncountable cardinal there exist a natural number $k$ and a subset $I^{\prime}$ of $I$ of power $\Lambda^{+}$such that every $a(i), i \in I^{\prime}$, has exactly $k$ elements $\alpha_{0}(i)<\alpha_{1}(i)<$ $\cdots<\alpha_{k-1}(i)$. For every $j<k$, the order-type of the set $\left\{\alpha_{j}(i), i \in I^{\prime}\right\}$ is at least $\Lambda^{+}$. In $k$ steps using the regularity of $\Lambda^{+}$, we can get a subset $J$ of $I^{\prime},|J|=\Lambda^{+}$, with a "coherent" ordering of all columns, i.e. such that for every two elements $i, i^{\prime}$ of $J$ and every $j<k$, we have

(5) $\alpha_{j}(i)<\alpha_{j}\left(i^{\prime}\right)$ iff $\alpha_{0}(i)<\alpha_{0}\left(i^{\prime}\right)$.

We may identify the elements of $J$ with ordinals less than $\Lambda^{+}$and construct an increasing sequence $\left\langle\beta(\gamma), \gamma<\Lambda^{+}\right\rangle$of elements of $J$ such that

(6) $a(\beta(\gamma)) \cap b(\beta(\delta)) \neq 0$ whenever $\gamma<\delta<\Lambda^{+}$.

To do that, it suffices to put

$$
\begin{aligned}
& \beta(0)=0, \\
& \beta(\gamma)=\sup \{\beta(\delta)+1, \delta<\gamma\} \text { if } \gamma \text { is a limit ordinal, } \\
& \beta(\gamma+1)=\sup \{\delta+1, \delta=\beta(\gamma) \vee b(\beta(\gamma)) \cap a(\delta) \neq 0\} .
\end{aligned}
$$

Since $a(\delta)$ 's are pairwise disjoint and the power of $b(\beta(\gamma))$ is less than $\Lambda^{+}$, it follows that $\beta(\gamma+1)$ is less than $\Lambda^{+}$, too and that the definition of $\beta(\gamma)$ is correct for every $\gamma<\Lambda^{+}$.

Now, it is easy to derive a contradiction. Let $\delta=\omega^{\mu+1}$ (ordinal power). For all $s<k$, let

$$
\Gamma_{s}=\left\{\gamma<\omega^{\mu+1}: \alpha_{s}(\beta(\gamma)) \in b(\beta(\delta)) .\right.
$$

Then by (6), $\omega^{\mu+1}=\Gamma_{0} \cup \cdots \cup \Gamma_{k-1}$. It follows that some $\Gamma_{s}$ has order-type $\omega^{\mu+1} \geqslant \mu+1$ contradicting (4). Hence $|I| \leqslant \Lambda$ and the proof is complete.

1. Basic construction. Given a regular uncountable cardinal $\kappa$, we shall construct a Boolean algebra of power $\kappa$ with no rigid or homogeneous factors. Let $\boldsymbol{A}$ be a free Boolean algebra with $\kappa$ free generators $a(\alpha), \alpha<\kappa$. For every subset $s$ of $\kappa$, let $A(s)$ denote the subalgebra of $A$ generated by $a(\alpha), \alpha \in s$. Hence $A=A(\kappa)$ and every element of $A$ belongs to some $A(s)$ for a finite $s$. The algebra $A$ can be alternatively described as follows. Let $F$ be the set of all mappings from finite subsets of $\kappa$ to the two element set $\{-1,1\}$. Let $\leqslant_{F}$ be the partial ordering of $F$ defined by reversed inclusion i.e. $f \leqslant_{F} g$ iff $g \subseteq f$. The complete Boolean algebra determined by the partially ordered set $\left(F, \leqslant_{F}\right)$ is isomorphic to the completion $A^{c}$ of $A$. We can identify every $a(\alpha)$ with a mapping from $F$ whose domain is $\{\alpha\}$ and the only value is 1 . Then $F$ is a dense subset of $A$ and $\leqslant_{F}$ coincides with the canonical ordering of $A$. The algebra $B$ we are constructing is a quotient of $A$ modulo a suitable ideal. To define the ideal, let $W$ be the stationary subset of $\kappa$ defined as follows.

$$
W=\left\{\alpha<\kappa, \operatorname{cf}(\alpha)=\boldsymbol{\kappa}_{0} \text { and } \alpha \text { is divisible by }|\alpha|\right\} .
$$

To every $\alpha \in W$ choose an increasing sequence $\xi(\alpha, n), n<\omega$, of odd ordinals whose limit is $\alpha$ such that for every $\beta, \beta<\kappa$,

$$
\{\alpha \in W, \xi(\alpha, 0)>\beta\} \text { is stationary in } \kappa \text {. }
$$

Let $I$ be the ideal on $A$ generated by the set $\{a(\alpha)-a(\xi(\alpha, n)), \alpha \in W, n<\omega\}$. Let $B$ be the quotient $A / I$ and for every $u \in A$, let $[u]$ denote the equivalence class 
of $u$. For each subset $s$ of $\kappa$, let $B(s)$ denote the set of all $[u], u \in A(s)$. Let $F / I$ denote the set of all elements [ $f$ ] $f \in F$, hence $F / I$ is a dense subset of $B$. It is easy to see that the power of $B$ and of every one of its factor is $\kappa$. First, we shall show that $B$ and hence $B^{c}$ has no rigid factors.

LeMma 1. For every nonzero $u, B \uparrow u$ is not rigid.

Proof. Let $s$ be a finite subset of $\kappa$ such that $u$ belongs to $B(s)$. Let $\eta, \eta<\kappa$, be an even nonlimit ordinal bigger than every element of $s$. Then $\eta$ belongs neither to $W$ nor to a sequence $\xi(\alpha, n)$. If $g$ is the automorphism of $A$ identical on every $a(\alpha)$, $\alpha \neq \eta$ satisfying $g(a(\eta))=-a(\eta)$ then $g$ maps $A(s)$ onto itself and determines a nontrivial automorphism of $B \uparrow u$.

In the rest of this section, we shall prove that $B^{c}$ and hence $B$ has no homogeneous factors. We shall prove some properties of algebras $B$ and $B(\alpha), \alpha<\kappa$.

LEMMA 2. For every $\alpha, \alpha \in W$, we have

(i) $[a(\alpha)]=\wedge\{[a(\xi(\alpha, n))] ; n<\omega\}$.

(ii) $\wedge\{[a(\alpha)], \alpha \in s\}=0_{B}$ for every infinite $s \subseteq W$.

(iii) For every element $u$ of $F / I$ disjoint from $[a(\alpha)]$, there is $\bar{u}$ in $B(\alpha)$ such that

(9) $v \geqslant u$ iff $v \geqslant \bar{u}$ holds for every $v \in B(\alpha)$.

If $\alpha$ does not belong to $W$, the same holds for every $u$. Hence $\bar{u}$ is the least element of $B(\alpha)$ bigger than $u$.

Proof. (i) It follows from the definition of $I$ that $[a(\alpha)] \leqslant[a(\xi(\alpha, n))]$ holds for every $n$. This gives the $\leqslant$ inequality. To prove the other inequality, let $f \in F$ be such that $[f]$ is less than all elements on the right-hand side of (i) and disjoint from $[a(\alpha)]$. We may assume that $\alpha$ belongs to the domain of $f$. If $f(\alpha)=1$, then $[f]$ must be zero. Assume that $f(\alpha)=-1$. As the domain of $f$ is finite, there is a natural number $n$ such that $\xi(\alpha, n)$ belongs neither to $\operatorname{dom}(f)$ nor to any sequence $\xi(\beta, m)$, where $\beta \in W \cap \operatorname{dom}(f)$ and $\alpha \neq \beta$. Let $g$ extend $f$ by $g(\xi(\alpha, n))=-1$. Note that $g$ belongs to $I$ iff $f$ belongs to $I$. Since $g$ is disjoint from $a(\xi, n)$ and $g \leqslant f$, we have $g \in I$. Hence $[f]$ must be zero and the other inequality is proved.

(ii) Let $f \in F$ be such that $[f]$ is less than every $[a(\alpha)], \alpha \in s$. We shall show that [ $f$ ] must be zero. As the domain of $f$ is finite, there is an $\alpha \in s$ which does not belong to $\operatorname{dom}(f)$. If we extend $f$ to a mapping $g$ by $g(\alpha)=-1$, then $g \leqslant f$ and $g$ is disjoint from $a(\alpha)$. Hence $[g]$ must be zero according to our assumption on $[f]$.

On the other hand, it follows from the definition of $g$ that $g \in I$ iff $f \in I$. Hence $[f]$ must be zero, too. This completes the proof of (ii).

To prove (iii), note that the case $u=0_{B}$ is trivial. Assume that $u$ is a nonzero element of $F / I, u=[f]$ for some $f$. If we put $f^{\prime}=f \nmid \alpha$ then $\left[f^{\prime}\right]$ belongs to $B(\alpha)$ and $\left[f^{\prime}\right] \geqslant u$. Note that for every $\beta \in W$ such that $f(\beta)=1$, we have $[a(\beta)] \geqslant[f]$ and, consequently, $[a(\xi(\beta, n))] \geqslant[f]$ holds for every $n<\omega$. Since $u$ is disjoint from $[a(\alpha)]$, either $\alpha$ does not belong to the domain of $f$ or $f(\alpha)=-1$. Consequently, if we have $f(\beta)=1$ for some $\beta \in W$ then either $\beta<\alpha$ or $\beta>\alpha$ (if $\alpha$ does not belong to $W$, the same holds for every [ $f]$ ). If $\beta<\alpha$, we already have it in the domain of $f^{\prime}$ and if $\beta>\alpha$ some, but only finitely many, ordinals $\xi(\beta, n)$ may fall below $\alpha$. We 
extend $f^{\prime}$ to $g$ putting $g(\xi(\beta, n))=1$ for every such $\xi(\beta, n)$. For any such $\xi(\beta, n)$ we cannot have $f^{\prime}(\xi(\beta, n))=-1$ for $f$ was nonzero, hence $g$ is a mapping from $F$. If we take $[g]$ for $\bar{u}$, we have (9). This completes the proof.

We shall define a hierarchy $Q(\alpha), \alpha<\kappa$, of subsets of $B^{c}$ in analogy to hierarchy $B(\alpha)$. For every $\alpha, \alpha<\kappa$, let

$$
Q(\alpha)=\left\{u \in B^{c}, u=\vee a \text { for some } a \subseteq B(\alpha)\right\} .
$$

It is clear from the definition that every $Q(\alpha)$ is closed under arbitrary sums. It turns out however that $Q(\alpha)$ for $\alpha \in W$ need not be closed under complements and hence $Q(\alpha)$ is not a subalgebra of $B^{c}$. Note that the statement (iii) of Lemma 2 generalizes to the case where $B(\alpha)$ is replaced by $Q(\alpha)$. The following is a simple consequence of this fact.

Corollary 3. For every $\alpha \in W$, and every subset a of $Q(\alpha)$, if $\wedge a$ is disjoint from $[a(\alpha)]$ then it belongs to $Q(\alpha)$. The same holds for every subset of $Q(\alpha)$ provided that $\alpha$ does not belong to $W$.

Proof. We shall first generalize the statement (iii) of Lemma 2 as follows: For every $\alpha \in W$ and every $u \in B^{c}$ disjoint from $[a(\alpha)]$, there is some $\bar{u} \in Q(\alpha)$ such that $\bar{u} \geqslant u$ and (9) holds for every $v$ in $Q(\alpha)$.

To prove this, let $u$ be the join of a family $u_{j}, j \in J$, of elements of $F / I$. For every $j \in J$ let $\bar{u}_{j}$ be as in (iii) of Lemma 2. If $\bar{u}$ is the join of all $\bar{u}_{j}, j \in J$, then $\bar{u}$ belongs to $Q(\alpha)$ and satisfies (9) for every $v$ in $Q(\alpha)$.

Now, let $a$ be a subset of $Q(\alpha)$ such that $\wedge a$ is disjoint from $[a(\alpha)]$. If $u$ is the meet of $a$ and we take $\bar{u}$ with the above property, then $\bar{u}$ belongs to $Q(\alpha)$ and $\bar{u}$ is less than all elements of $a$ according to (9). Hence $\bar{u}$ is the meet of $a$ and $\wedge a$ belongs to $Q(\alpha)$.

If $\alpha \notin W$, it is easy to see that the same holds for every subset of $Q(\alpha)$.

We need a bound on the cardinality of antichains in $B$ to show that every element of $B^{c}$ belongs to some $Q(\alpha)$.

Lemma 4. B satisfies the countable antichain condition.

Proof. Suppose that there is an uncountable antichain $a$ in $B$. We may assume that every element of $a$ is nonzero and belongs to $F / I$. Thus for every $u \in a$ there is a unique $f, f \in F$ such that $u=[f]$. Let $F_{0}=\{f,[f] \in a\}$. As the domain of every $f$ is finite, it follows from the well-known theorem of Erdös and Rado [3] that there is a finite set $s$ and a subset $F_{1}$ of $F_{0},\left|F_{1}\right|=\left|F_{0}\right|$ such that $\operatorname{dom}(f) \cap \operatorname{dom}(g)=s$ holds for every two different $f, g$ from $F_{1}$. We may assume as well that

$$
f \nmid s=g \nmid s \text { for every pair } f, g \text { from } F_{1} \text {. }
$$

Hence $F_{1}$ is a set of pairwise compatible mappings. It follows from our assumption on $a$ that $[f],[g]$ are disjoint in $B$, thus we have the following

for every two different mappings $f, g$ from $F_{1}$, there exist $\alpha \in W$ and $n<\omega$ such that $f(\xi(\alpha, n))=-1$ and $g(\alpha)=1$ or vice versa. 
It follows from (10) that neither $\alpha$ nor $\xi(\alpha, n)$ from (11) belong to $s$ for otherwise [ $f$ ] or $[g]$ would be zero. We may thus leave out the whole $s$ from the domains of mappings in $F_{1}$ and assume that the domains are pairwise disjoint.

For every $f \in F_{1}$, let

$$
\begin{gathered}
A_{f}=\{\xi(\alpha, n), f(\xi(\alpha, n))=-1\}, \\
B_{f}=\{\xi(\alpha, m), f(\alpha)=1, m<\omega\} .
\end{gathered}
$$

Then every $A_{f}$ is finite and the order-type of every $B_{f}$ is less than $\omega \cdot \omega$. It follows from (11) that the families of sets $\left\{A_{f}, f \in F_{1}\right\}$ and $\left\{B_{f}, f \in F_{1}\right\}$ satisfy the assumptions of the Proposition from $\S 0$, and accordingly, $F_{1}$ must be countable-a contradiction.

We are ready to show that $B^{c}$ has no homogeneous factors.

LEMma 5. For every nonzero element $u$ from $B^{c}, B^{c} \uparrow u$ is not homogeneous.

Proof. Suppose that $B^{c}$ has a homogeneous factor $B^{c} \uparrow u$. We may assume that $u$ belongs to $B$ and hence to $B(s)$ for some finite subset $s$ of $\kappa$. It follows from (8) that the set

$$
W^{\prime}=\{\alpha \in W, \xi(\alpha, 0) \text { is above every element of } s\}
$$

is stationary in $\kappa$. There is a stationary subset $W^{\prime \prime}$ of $W^{\prime}$ and an ordinal $\eta$ such that

$$
\xi(\alpha, 0)=\eta \quad \text { for every } \alpha \in W^{\prime \prime}
$$

since $\xi(\alpha, 0)$ is a regressive function of $\alpha$ on $W^{\prime}$. If we put $v=u \wedge[a(\eta)]$ and $w=u \wedge-[a(\eta)]$ then $v, w$ are disjoint nonzero elements of $B \uparrow u$. Suppose that $G$ is an automorphism of $B^{c} \uparrow u$ which moves $v$ onto $w$. We may extend $G$ by identity outside of $u$ to an automorphism of $B^{c}$. It turns out that

$$
X_{G}=\{\alpha<\kappa, G \text { maps } Q(\alpha) \text { into } Q(\alpha)\} \text { is closed unbounded in } \kappa,
$$

and

$$
S=W^{\prime \prime} \cap X_{G} \text { is stationary in } \kappa .
$$

For every $\alpha \in S, v \wedge[a(\alpha)]$ is nonzero and we shall show that $G$ maps it into $Q(\alpha)$. According to Lemma 2(i), we have

$$
v \wedge[a(\alpha)]=v \wedge \wedge\{[a(\xi(\alpha, n))] ; n<\omega\}
$$

Note that all elements on the right-hand side of (15) belong to $Q(\alpha)$. Hence

$$
G(v \wedge[a(\alpha)])=w \wedge \wedge\{G([a(\xi(\alpha, n))]) ; n<\omega\}
$$

and every element on the right-hand side belongs again to $Q(\alpha)$ according to (13) and (14). It follows from (12) that $w$ is disjoint from $[a(\alpha)]$ and, according to Corollary 3, $G(v \wedge[a(\alpha)])$ is a nonzero element of $Q(\alpha)$ for every $\alpha \in S$. We shall need one more fact about the hierarchy of $B(\alpha)$ 's. As every $\alpha \in W$ is divisible by $|\alpha|$, there is a mapping $\varphi$ from $\kappa$ onto $B$ such that

$$
\varphi \text { maps every } \alpha \in W \text { onto } B(\alpha) \text {. }
$$

For every $\alpha \in S$, let $h(\alpha)$ be the least ordinal such that $\varphi(h(\alpha))$ is nonzero and less than $G(v \wedge[a(\alpha)])$. It follows from (17) that $h$ is regressive on $S$ and hence constant 
on a stationary subset $S^{\prime}$ of $S$. It follows from Lemma 2(ii) that $v \wedge \wedge\{[a(\alpha)]$; $\left.\alpha \in S^{\prime}\right\}$ is zero but we have shown that $G$ maps it onto a nonzero element of $B^{c}-$ a contradiction. This completes the proof of Lemma 5 and shows that $B$ is a Boolean algebra of power $\kappa$ with no rigid or homogeneous factors and that $B^{c}$ has no rigid or homogeneous factors, too.

2. Isomorphism types. It is easy to see that every Boolean algebra with no homogeneous factors is atomless. All countable atomless Boolean algebras are isomorphic and hence homogeneous. Consequently, there are no countable Boolean algebras with no rigid or homogeneous factors. We are going to show that for every uncountable cardinal $\kappa$ there are $2^{\kappa}$ isomorphism types of Boolean algebras of power $\kappa$ with no rigid or homogeneous factors. To get this result, we shall modify the basic construction using some ideas of Monk and Rassbach [9].

THEOREM 1. For every uncountable regular cardinal $\kappa$, there are Boolean algebras $B_{\alpha}, \alpha<2^{\kappa}$, of power $\kappa$ such that for every $\alpha, \beta<2^{\kappa}$, we have

(i) $B_{\alpha}$ and $B_{\alpha}^{c}$ have no rigid or homogeneous factors,

(ii) $B_{\alpha}^{c}$ and $B_{\beta}^{c}$ are not isomorphic for distinct $\alpha, \beta$, and

(iii) $B_{\alpha}$ satisfies the countable antichain condition.

Hence if $\kappa^{\aleph_{0}}=\kappa$, we have $2^{\kappa}$ isomorphism types of complete Boolean algebras of power $\kappa$ with no rigid or homogeneous factors.

Proof. Given an uncountable regular cardinal $\kappa$, we shall construct a Boolean algebra $B(X)$ of power $\kappa$ for every nonempty $X, X \subseteq \kappa$. It suffices to modify properly the definition of the ideal $I$ used in the basic construction. Let $W$ be as in (7) and let $W_{\gamma}, \gamma<\kappa$, be pairwise disjoint stationary subsets of $W$. For every $\gamma$ and every $\alpha \in W_{\gamma}$ choose an increasing sequence of odd ordinals $\xi(\alpha, n), n<\omega$, with limit $\alpha$ such that (8) holds for every $W_{\gamma}, \gamma<\kappa$. For every nonempty $X \subseteq \kappa$, put $W(X)=\cup\left\{W_{\gamma}, \gamma \in X\right\}$. As above, let $A$ be the free Boolean algebra with $\kappa$ free generators $a(\alpha), \alpha<\kappa$, and let $I(X)$ be the ideal on $A$ generated by elements $a(\alpha)-a(\xi(\alpha, n))$ for $\alpha \in W(X), n<\omega$. Let $B(X)$ denote the quotient $A / I(X)$. Now it is easy to prove the analogues of Lemmas 1-5 from the preceding section. Hence we have (i) and (iii) from Theorem 1. It remains to prove (ii). Let $X, Y$ be nonempty subsets of $\kappa$ such that

there is a $\gamma, \gamma \in X-Y$.

Suppose that there is an isomorphism $G$ which maps $B(X)^{c}$ onto $B(Y)^{c}$. For every $\alpha<\kappa$, let

$$
\begin{aligned}
& B(X, \alpha)=\{[u] \in B(X), u \in A(\alpha)\} \\
& Q(X, \alpha)=\left\{v \in B(X)^{c}, v=\vee a \text { for some } a \subseteq B(X, \alpha)\right\}
\end{aligned}
$$

Then

(19) $X_{G}=\{\alpha<\kappa, G$ maps $Q(X, \alpha)$ into $Q(Y, \alpha)\}$ is closed unbounded in $\kappa$.

It follows from (18) that $W_{\gamma}$ is a subset of $W(X)$ disjoint from $W(Y)$. Hence

$$
S=X_{G} \cap W_{\gamma} \text { is a stationary subset of } W(X) \text { disjoint from } W(Y) \text {. }
$$


According to Lemma 2(i), we have

$$
G([a(\alpha)])=\wedge\{G([a(\xi(\alpha, n))]) ; n<\omega\} \quad \text { for } \alpha \in S
$$

and it follows from (19) that all elements on the right-hand side belong to $Q(Y, \alpha)$. Hence $G([a(\alpha)])$ belongs to $Q(Y, \alpha)$ according to Corollary 3 and (20). As in $\S 1$, let $\varphi_{Y}$ be a mapping of $\kappa$ onto $B(Y)$ such that

$$
\varphi_{Y} \text { maps } \alpha \text { onto } B(Y, \alpha) \text { whenever } \alpha \in W(Y) \text {. }
$$

For every $\alpha \in S$, let $h(\alpha)$ be the least ordinal such that $\varphi(h(\alpha))$ is nonzero and less than $G([a(\alpha)])$. It follows from (21) that $h$ is regressive on $S$ and hence constant on some stationary $S^{\prime} \subseteq S$. It follows that the set of all $G([a(\alpha)])$ for $\alpha \in S^{\prime}$ has a nonzero lower bound in $B(Y)^{c}$ which contradicts Lemma 2(ii). Hence $B(X)^{c}$ and $B(Y)^{c}$ are not isomorphic.

Since every $B(X)$ satisfies the countable antichain condition, the power of $B(X)^{c}$ is $\kappa^{\aleph_{0}}$. This concludes the proof of Theorem 1 .

THEOREM 2. For every singular cardinal $\kappa$, there are $2^{\kappa}$ nonisomorphic (noncomplete) Boolean algebras of power $\kappa$ with no rigid or homogeneous factors.

Proof. Let $\kappa$ be a singular cardinal and $\left\langle\Lambda_{\alpha}, \alpha<\operatorname{cf}(\kappa)\right\rangle$ be an increasing sequence of regular uncountable cardinals with limit $\kappa$. For every $\alpha<\operatorname{cf}(\kappa)$, let $B(\alpha, \beta), \beta<2^{\Lambda_{\alpha}}$, be a sequence of Boolean algebras of power $\Lambda_{\alpha}$ constructed in Theorem 1 .

For every $f$ from the cartesian product $P_{\alpha<\operatorname{cf}(\kappa)} 2^{\Lambda_{\alpha}}$, let $B_{f}$ be the product of algebras $B(\alpha, f(\alpha)), \alpha<\operatorname{cf}(\kappa)$. Note that $B_{f}$ has power greater than $\kappa$ and that every element of it is a sequence $\left\langle u_{\alpha}, \alpha<\operatorname{cf}(\kappa)\right\rangle$, where $u_{\alpha} \in B(\alpha, f(\alpha))$ for every $\alpha<$ $\operatorname{cf}(\kappa)$. Let $J_{f}$ be the ideal of all $u \in B_{f}$ such that only finitely many $u_{\alpha}$ 's are nonzero. It is easy to see that $J_{f}$ has power $\kappa$ and that the set of all elements of $J_{f}$ and of all complements of elements of $J_{f}$ is a subalgebra of $B_{f}$. We shall call it $C_{f}$. Every $C_{f}$ has no rigid or homogeneous factors. To complete the proof, it remains to show that $C_{f}$ and $C_{g}$ are not isomorphic provided that $f, g$ are distinct. We shall use the fact that for every $\alpha<\operatorname{cf}(\kappa), \beta<2^{\Lambda_{\alpha}}$, every factor of $B(\alpha, \beta)$ has power $\Lambda_{\alpha}$.

Let $f(\alpha) \neq g(\alpha)$ for some $\alpha$. Suppose that $G$ is an isomorphism which maps $C_{f}$ onto $C_{g}$. Let $u$ be the element of $C_{f}$ such that $u_{\beta}$ is zero for every $\beta \neq \alpha$ and $u_{\alpha}$ is the unit element of $B(\alpha, f(\alpha))$. In other words, $u$ is the largest element of $C_{f}$ such that every factor of $C_{f} \uparrow u$ has power $\Lambda_{\alpha}$. Thus $G(u)$ is the largest element of $C_{g}$ with the same property, we shall denote it by $v$. It turns out that $v_{\alpha}$ is the unit of $B(\alpha, g(\alpha))$, the other components of $v$ being zero. If we restrict $G$ to $C_{f} \uparrow u$, we get an isomorphism of $B(\alpha, f(\alpha))$ and $B(\alpha, g(\alpha))$-a contradiction.

Note that $\operatorname{sat}\left(C_{f}\right)=(\operatorname{cf}(\kappa))^{+}$for every $f$, and that no $C_{f}$ is complete.

Problem. It remains open whether there is a complete Boolean algebra with no rigid or homogeneous factors and with power $\kappa$ for every singular cardinal $\kappa$, $\kappa^{\kappa_{0}}=\kappa$.

Rubin [11] asked whether there are Boolean algebras with no homogeneous factors satisfying some weaker homogeneity property. It turns out that the existence of weakly homogeneous complete Boolean algebras with no homogeneous factors is 
ruled out by the following (unpublished) result due to Solovay which was proved independently by Koppelberg [5].

TheOREM (Solovay, KopPelBerG). Every complete weakly homogeneous Boolean algebra is a power of some complete homogeneous Boolean algebra.

Hence every complete weakly homogeneous Boolean algebra has a homogeneous factor. The completion of every Boolean algebra constructed in Theorem 1 has no homogeneous factor. It follows from the above theorem that Boolean algebras we have constructed so far are not weakly homogeneous.

Problem. It remains open whether there are noncomplete weakly homogeneous Boolean algebras with no homogeneous factors.

It is possible to construct Boolean algebras with no homogeneous factors in which every element, which is not zero or unit, is moved by some automorphism.

3. Embeddings preserving saturatedness. We shall show that the class of Boolean algebras with no rigid or homogeneous factors is universal with respect to embeddings, namely, we shall show that every Boolean algebra can be completely embedded in a complete Boolean algebra with no rigid or homogeneous factors. These embeddings have two interesting properties: Every automorphism of the smaller algebra can be extended to an automorphism of the larger algebra and both algebras have the same saturatedness except the trivial case when the smaller algebra is finite. We shall compare this result with embedding theorems due to Kripke [6] and Koppelberg [5].

THEOREM 3. Every Boolean algebra $C$ can be completely embedded in a complete Boolean algebra $D$ without homogeneous or rigid factors and with the following properties.

(i) Every automorphism of $C$ can be extended to an automorphism of $D$,

(ii) $\operatorname{sat}(C)=\operatorname{sat}(D)$ whenever $C$ is infinite.

Proof. We shall assume that $C$ is complete, otherwise we take $C^{c}$ instead of $C$. Let $\kappa$ be the cardinality of $C$ whenever it is infinite, otherwise put $\kappa=\boldsymbol{\aleph}_{0}$. If we put

$$
W=\left\{\alpha<\kappa^{+}, \operatorname{cf}(\alpha)=\omega \text { and } \alpha \text { is divisible by } \kappa\right\}
$$

then $W$ is stationary in $\kappa^{+}$.

Let $B$ be an algebra of power $\kappa^{+}$constructed from $W$ and appropriate sequences $\xi(\alpha, n), n<\omega$, by the method described in $\S 1$. If $D$ is the direct sum $C \oplus B$, then $D$ is complete and $B, C$ are completely embedded in $D$. It is clear from the definition of direct sum that every automorphism $f$ of $C$ extends to an automorphism of $D$. Namely, if we put $g(u, v)=(f(u), v)$ for every pair $(u, v)$ from the dense subset of $D$ used in the definition of direct sum, then $g$ is an automorphism of the dense subset with respect to the canonical ordering and $g$ extends uniquely to an automorphism of $D$ with $g(u)=f(u)$ for every $u$ from $C$.

We shall show that $D$ has no rigid or homogeneous factors and that $\operatorname{sat}(D)=\boldsymbol{\aleph}_{1}$. $\operatorname{sat}(C)$. Let $A, I, F, a(\alpha), B(\alpha)$ for $\alpha<\kappa^{+}$be as in $\S 1$. Note that $d_{F}=C \times F / I$ is dense in $D$. If 0 denotes the empty mapping, we can identify every nonzero $u \in C$ 
with the pair $(u, 0)$ from $d_{F}$ and every $[f] \in F / I$ with $\left(1_{C},[f]\right)$. No element of $d_{F}$ determines a rigid factor of $D$ since every automorphism of $B$ extends to an automorphism of $D$ and we can modify the argument of Lemma 1 . Hence $D$ has no rigid factors.

For every $\alpha<\kappa^{+}$, let $D(\alpha)=C \times B(\alpha)$ and

$$
R(\alpha)=\{u \in D, u \text { is a join of a subset of } D(\alpha)\} \text {. }
$$

It is easy to prove (i) and (ii) of Lemma 2 for $D$. If we replace $F / I, Q(\alpha)$ in turn by $d_{F}, R(\alpha)$, we obtain (iii) from Lemma 2 and Corollary 3.

The following result is the counterpart of Lemma 4 and gives (ii) of Theorem 3.

Lemma 6. $\operatorname{sat}(D)=\aleph_{1} \cdot \operatorname{sat}(C)$.

Proof. Let $\mu$ denote the cardinal on the right-hand side and let $a$ be an antichain in $D,|a|=\mu$. We may assume that $a$ is a subset of $d_{F}$ and define $F_{0}$ as

$$
F_{0}=\{f \in F,(u,[f]) \in a \text { for some } u\} .
$$

Note that $\left|F_{0}\right|=\mu$ for otherwise there would be an antichain of power $\mu$ in $C$. Let $F_{1}$ be a subset of $F_{0}$ obtained from $F_{0}$ as in the proof of Lemma 4 . Hence $F_{1}$ has power $\mu$ and we may assume that the domains of $F_{1}$ are pairwise disjoint. Let $A_{f}, B_{f}$, $f \in F_{1}$, be defined as in the proof of Lemma 4. Since $\mu$ is regular and uncountable and every $A_{f}$ is finite, there is a natural number $k$ and a subset $F_{2}$ of $F_{1}$ of power $\mu$ such that every $A_{f}, f \in F_{2}$, consists of exactly $k$ ordinals $\alpha_{0}(f)<\alpha_{1}(f)<\cdots<$ $\alpha_{k-1}(f)$. As in the proof of the Proposition in $\S 0$, there is a subset $F_{3}$ of $F_{2}$, $\left|F_{3}\right|=\mu$, such that

(23) $\left\{\alpha_{0}(f), f \in F_{3}\right\}$ has order-type $\mu$, and

(24) for every $j<k, f, f^{\prime} \in F_{3}$, we have

$$
\alpha_{j}(f)<\alpha_{j}\left(f^{\prime}\right) \text { iff } \alpha_{0}(f)<\alpha_{0}\left(f^{\prime}\right) .
$$

It follows from (23) that $F_{3}$ is well-ordered, let $f_{\beta}, \beta<\mu$, be the corresponding enumeration. For every $\beta<\mu$, choose a $u_{\beta}$ such that $\left(u_{\beta}\right.$, $\left.\left[f_{\beta}\right]\right)$ belongs to $a$. It turns out that if $\beta<\gamma<\mu$ then either

(25) $u_{\beta}, u_{\gamma}$ are disjoint in $C$, or $\left[f_{\beta}\right],\left[f_{\gamma}\right]$ are disjoint in $B$.

In what follows, we shall write simply $A_{\beta}, B_{\beta}$ instead of $A_{f_{\beta}}, B_{f_{\beta}}$. It follows from the fact that any two mappings from $F_{3}$ are compatible that the last condition is equivalent to

$$
A_{\beta} \cap B_{\gamma} \neq 0 \quad \text { or } \quad A_{\gamma} \cap B_{\beta} \neq 0 .
$$

To complete the proof, we shall construct an increasing sequence

$$
\left\langle\eta_{\alpha}, \alpha<\mu\right\rangle
$$

of ordinals less than $\mu$ such that

(28) for every $\beta, \gamma, \beta<\gamma<\mu$, there is an ordinal $\delta, \eta_{\beta} \leqslant \delta<\eta_{\beta+1}$ such that $A_{\delta} \cap B_{\eta_{\gamma}} \neq 0$. Let $\eta_{0}=0$. If we have defined $\eta_{\alpha}$, the set $\left\{\beta, \eta_{\alpha}<\beta<\mu\right\}$ splits into two parts according to (25), (26). Let $U_{\alpha}$ consist of all $\beta$ such that $\beta>\eta_{\alpha}$ and $\eta_{\alpha}, \beta$ do not satisfy (26). Choose a maximal subset $V_{\alpha}$ of $U_{\alpha}$ such that every couple of elements of $V_{\alpha}$ does not satisfy (26). Then the cardinality of $V_{\alpha}$ is less than $\mu$ since 
$\left\{\mu_{\xi}, \xi \in V_{\alpha}\right\}$ is an antichain in $C$. Let $\nu_{\alpha}$ be an upper bound for $V_{\alpha}$, hence for every $\gamma>\nu_{\alpha}$ there is a $\delta, \eta_{\alpha} \leqslant \delta<\nu_{\alpha}$ such that $\gamma, \delta$ satisfy (26). But all $A_{\gamma}$ 's are nonempty and pairwise disjoint and the union of all $B_{\delta}, \eta_{\alpha} \leqslant \delta<\nu_{\alpha}$, has power less than $\mu$. Hence there is an $\varepsilon, \varepsilon<\mu$, such that $A_{\gamma} \cap B_{\delta}=0$ for every $\gamma \geqslant \varepsilon$ and every $\delta$, $\eta_{\alpha} \leqslant \delta<\nu_{\alpha}$. Let $\eta_{\alpha+1}$ be the least such $\varepsilon$. Then for every $\gamma, \eta_{\alpha+1} \leqslant \gamma$, there is some $\delta, \eta_{\alpha} \leqslant \delta<\eta_{\alpha+1}$, such that $A_{\delta} \cap B_{\gamma} \neq 0$.

For a limit ordinal $\Lambda$, let $\eta_{\Lambda}$ be the supremum of all $\eta_{\alpha}, \alpha<\Lambda$. It is clear from the definition that the sequence (27) satisfies (28).

Now, choose $\gamma, \gamma>\omega \cdot \omega$. It follows from (28) that there is an increasing sequence of ordinals $\delta_{\beta}, \beta<\gamma$, such that $B_{\eta_{\gamma}}$ has a common element with every $A_{\delta_{\beta}}, \beta<\gamma$. But the order-type of $B_{\eta_{\gamma}}$ is less than $\omega \cdot \omega$, and it follows from (23), (24) that it is too small to satisfy the last requirement - a contradiction. This completes the proof of Lemma 6.

It follows from the above lemma and from the definition of $\kappa$ that $D$ satisfies the $\kappa^{+}$-antichain condition, hence $D$ is the union of all subsets $R(\alpha), \alpha<\kappa^{+}$. Note also that there is a mapping $\varphi$ which maps every $\alpha \in W$ onto $D(\alpha)$. An obvious modification of the proof of Lemma 5 shows that $D$ has no homogeneous factors. This completes the proof of Theorem 3 .

REMARKS. It was shown in [14] that every atomless Boolean algebra $C$ can be completely embedded in a complete Boolean algebra $D$ with no rigid or homogeneous factors in such a way that every element of $C$ was left fixed by every automorphism of $D$. Hence no nontrivial automorphism of $C$ (if any) could be extended to an automorphism of $D$. Note that (i) of Theorem 3 gives just the opposite case.

It follows from Theorem 3 that the automorphism group of an arbitrary Boolean algebra is a subgroup of the automorphism group of some complete Boolean algebra with no rigid or homogeneous factors. If we return to the embeddings described in Theorem 3, it is natural to ask whether the automorphism group $\operatorname{Aut}(C)$ of $C$ is a proper subgroup of $\operatorname{Aut}(D)$. It turns out that the answer is affirmative in most cases if we restrict ourselves to complete algebras. A deep result due to Rubin [11] shows that every two complete Boolean algebras with no rigid factors have the same automorphism group iff the algebras are isomorphic. Hence if $C$ has no rigid factors, $\operatorname{Aut}(C)$ must be a proper subgroup of $\operatorname{Aut}(D)$ for $C$ and $D$ have different cardinalities.

Let us compare Theorem 3 with other embedding theorems. Kripke [6] showed that every Boolean algebra can be completely embedded in a complete homogeneous Boolean algebra. A similar result was proved by Koppelberg [5] by a different method. These embeddings need not preserve saturatedness. Balcar and the author proved in [14] that every infinite Boolean algebra can be completely embedded in a rigid complete Boolean algebra with the same saturatedness. It was shown there that the statement "every infinite Boolean algebra can be completely embedded in a homogeneous Boolean algebra with the same saturatedness" is not a theorem of ZFC. An easy modification of the argument shows that the statement "every infinite Boolean algebra can be completely embedded in a Boolean algebra with an atomless 
homogeneous factor and with the same saturatedness" also is not a theorem of ZFC. These results indicate that embeddings preserving saturatedness impose restrictions on the automorphism group of the larger algebra and that Theorem 3 is the strongest possible result in this direction.

4. Embeddings preserving distributivity. We shall prove that for every cardinal $\kappa$, there are complete Boolean algebras with no rigid or homogeneous factors and with a $\kappa$-closed dense subset. The main result of this section shows that every $\kappa$-distributive Boolean algebra can be completely embedded in a complete $\kappa$-distributive Boolean algebra with no rigid or homogeneous factors.

THEOREM 4. Let $\kappa, \Lambda$ be infinite cardinals such that

$$
\Lambda^{\left(2^{\mathrm{x}^{+}}\right)}=\Lambda .
$$

Then there is a complete Boolean algebra of power $\Lambda^{+}$with no rigid or homogeneous factors which has a $\mathrm{k}$-closed dense subset.

Corollary (GCH). Given an infinite cardinal $\kappa$, there is a complete Boolean algebra of power $\Lambda$ with no rigid or homogeneous factors and with a $\kappa$-closed dense subset for every regular cardinal $\Lambda, \kappa^{+++} \leqslant \Lambda$.

Proof of Theorem 4. We shall modify the basic construction to get a complete Boolean algebra with a $\kappa$-closed dense subset.

Let $F$ be the set of all mappings whose domains are subsets of $\Lambda^{+}$of power at most $\kappa$ with values $1,-1$. Let $A$ be the complete Boolean algebra determined uniquely by the set $F$ ordered by reversed inclusion. For every $\alpha, \alpha<\Lambda^{+}$, let $a(\alpha)$ denote the mapping with domain $\{\alpha\}$ and value 1. Let $F(\alpha)$ denote the set of all mappings from $F$ whose domain is a subset of $\alpha$.

If we put

$$
W=\left\{\alpha<\Lambda^{+}, \operatorname{cf}(\alpha)=\kappa^{+} \text {and } \alpha \text { is divisible by } \Lambda\right\},
$$

then $W$ is stationary in $\Lambda^{+}$and the cardinality of every $F(\alpha), \alpha \in W$, is $\Lambda$ since $\Lambda^{\kappa}=\Lambda$ follows from (29). Hence there is a mapping $\varphi$ from $\Lambda^{+}$onto $F$ such that

$$
\varphi \text { maps every } \alpha \in W \text { onto } F(\alpha) \text {. }
$$

For every $\alpha \in W$ choose an increasing sequence $\xi(\alpha, \beta), \beta<\kappa^{+}$, of odd ordinals with limit $\alpha$ such that

$$
\{\alpha \in W, \xi(\alpha, 0)>\beta\} \text { is stationary for every } \beta, \beta<\Lambda^{+} .
$$

Let $I$ be the $\kappa^{+}$-complete ideal on $A$ generated by all elements $a(\alpha)-a(\xi(\alpha, \beta)$, for $\alpha \in W, \beta<\kappa^{+}$. Hence $I$ contains every element of $A$ less than a join of at most $\kappa$ generators of $I$. Note that $F$ is not a subset of $I$. If $f$ is a mapping whose domain consists of limit ordinals, then for every $u \in I$, there is an extension $f^{\prime}$ of $f$ such that $f^{\prime}$ and $u$ are disjoint.

For every $f \in F$, let $[f]$ denote the equivalence class of $f$ in the quotient $A / I$. Let $F / I$ be the set of all $[f], f \in F$, with the partial ordering induced by $A / I$. Let $B$ be 
the complete Boolean algebra uniquely determined by $F / I$. Clearly, $F / I$ is a $\kappa$-closed dense subset of $B$. We shall show that $B$ satisfies the conditions stated in Theorem 4.

It is easy to show that $B$ has no rigid factors. We have the following version of Lemma 2.

Lemma 7. For every $\alpha, \alpha \in W$, we have

(i) $[a(\alpha)]=\bigwedge\left\{[a(\xi(\alpha, \beta))], \beta<\kappa^{+}\right\}$,

(ii) $\wedge\{[a(\beta)], \beta \in s\}$ is zero for every $s \subseteq W,|s| \geqslant \kappa^{+}$, and

(iii) for every $u \in F / I$ disjoint from $[a(\alpha)]$, there is $f \in F(\alpha)$ such that

$$
[g] \geqslant u \quad \text { iff }[g] \geqslant[f] \text { holds for every } g \in F(\alpha) \text {. }
$$

Proof. (i) and (ii) are easy and (iii) is proved by the same way as in Lemma 2 using regularity of $\kappa^{+}$.

If we put

$$
Q(\alpha)=\{u \in B, u \text { is the join of a subset of } F(\alpha) / I\},
$$

we get Corollary 3 as well.

Lemma 8. B satisfies the $\left(2^{\alpha^{+}}\right)^{+}$-antichain condition.

Proof. Let $a$ be an antichain in $B$. We may assume that $a$ is a subset of $F / I$ and that every element of $a$ is nonzero. Hence for every $u \in a$, there is some $f \in F$ such that $u=[f]$. Let $F_{0}$ be the set consisting of all such $f$ 's. It turns out that for every couple of elements $f_{1}, f_{2}$ of $F_{0}$ either $f_{1}, f_{2}$ are incompatible mappings or there exist $\alpha \in W$ and $\beta<\kappa^{+}$such that $f_{1}(\alpha)=1$ and $f_{2}(\xi(\alpha, \beta))=-1$ or vice versa. We shall extend every $f \in F_{0}$ to $f^{\prime}, f \subseteq f^{\prime}$, as follows: Put $f^{\prime}(\xi(\alpha, \beta))=1$ for every $\beta<\kappa^{+}$ whenever $\alpha \in W$ and $f(\alpha)=1$. Note that $f^{\prime}$ is a mapping for otherwise $[f]$ would be zero in $B$. Hence $F_{0}^{\prime}=\left\{f^{\prime}, f \in F_{0}\right\}$ is a set of pairwise incompatible two-valued mappings. The cardinality of the domain of every $f^{\prime}$ is at most $\kappa^{+}$, hence it follows from well-known results that the cardinality of $F_{0}^{\prime}$ is at most $2^{\kappa^{+}}$. To complete the proof of Lemma 8 it suffices to show that there is a one-one correspondence between $F_{0}$ and $F_{0}^{\prime}$. In fact, if for some $f, g \in F_{0}$, we have $f^{\prime}=g^{\prime}$ then $f, g$ are compatible mappings and $[f],[g]$ cannot be disjoint, hence $f=g$.

It follows from (29) that $2^{\kappa^{+}}<\Lambda$, hence $B$ is the union of all $Q(\alpha), \alpha<\Lambda^{+}$, and we can use a similar argument as in the proof of Theorem 1 to show that $B$ has no homogeneous factors. This completes the proof of Theorem 4.

Sketch of the proof of Corollary. Note that the existence of a mapping satisfying (31) was one of the important points in our construction. If we assume generalized continuum hypothesis and if $\Lambda$ is regular, $\Lambda>\kappa^{++}$, then

$$
W=\left\{\alpha<\Lambda, \operatorname{cf}(\alpha)=\kappa^{+}\right\}
$$

is stationary in $\Lambda$ and for every $\alpha \in W$, we have $|\alpha|^{\kappa}=|\alpha|$. Hence there is a mapping $\varphi$ which maps $\Lambda$ onto $F$ and satisfies (31). In fact $\Lambda>\kappa^{+}$would suffice for this, but we need $\Lambda>\kappa^{++}$to get the $\Lambda$-antichain condition. If we proceed as above we obtain a complete Boolean algebra of power $\Lambda$ with no rigid or homogeneous factors and with a $\kappa$-closed dense subset. 
We can use the method of $\S 2$ to get more isomorphism types.

COROLlary. Let $\kappa, \Lambda$ be as in Theorem 4 , then there are $2^{\left(\Lambda^{+}\right)}$isomorphism types of $\kappa$-distributive complete Boolean algebras of power $\Lambda^{+}$with no rigid or homogeneous factors.

- The following lemma is a simple conclusion to a theorem concerning Easton's forcing [1].

Lemma (EASTON). Let $B$ be a $\kappa$-distributive Boolean algebra and let $C$ be a complete $B o o l e a n$ algebra with a $\Lambda$-closed dense subset. If the cardinality of every antichain in $B$ is at most $\Lambda$, then the direct sum $B \oplus C$ is $\kappa$-distributive.

THEOREM 5. Let $\mu$ be a cardinal. Every $\mu$-distributive complete Boolean algebra can be completely embedded in a $\mu$-distributive complete Boolean algebra with no rigid or homogeneous factors.

Proof. Let $C$ be a $\mu$-distributive Boolean algebra, let $\kappa=\operatorname{sat}(C)$ and $\nu=|C|$. If we put $\Lambda=\nu^{\left(2^{\alpha^{+}}\right)}$then we have (29). Using Theorem 4, let $B$ be a complete Boolean algebra of power $\Lambda^{+}$with a $\kappa$-closed dense subset and with no rigid or homogeneous factors. It follows from Easton's lemma above that the direct sum $D=C \oplus B$ is $\mu$-distributive. We shall show that it has no rigid or homogeneous factors.

Let $F, W, I, a(\alpha), F(\alpha)$ for $\alpha<\Lambda^{+}$and $\xi(\alpha, \beta)$ for $\alpha \in W, \beta<\kappa^{+}$be as in the proof of Theorem 4. If we put $d_{F}=C \times F / I$ then $d_{F}$ is dense in $D$. It is easy to see that $D$ has no rigid factors. We have Lemma 7 for $D$ as well, in particular (iii) holds for every $u$ from $d_{F}$. If we put

$$
D(\alpha)=C \times F(\alpha) / I
$$

and

$$
R(\alpha)=\{u \in D, u \text { is a join of a subset of } D(\alpha)\},
$$

for every $\alpha<\Lambda^{+}$, we get a corresponding version of Corollary 3. It follows from the definition of $\Lambda$ that the cardinality of every $D(\alpha), \alpha \in W$ is $\Lambda$, hence there is a mapping $\varphi$ from $\Lambda^{+}$onto $d_{F}$ which maps every $\alpha \in W$ onto $D(\alpha)$. To prove that $D$ has no homogeneous factors, we need a bound on the saturatedness of $D$. The following lemma gives a sufficient one although not the best one in all cases.

Lemma 9. D satisfies the $\Lambda^{+}$-antichain condition.

Proof. Put $\eta=\Lambda^{+}$and suppose that there is an antichain $a$ of power $\eta$ in $D$. We shall follow the proof of Lemma 6 and construct a set $F_{0}, F_{0} \subseteq F$, of power $\eta$. Using a theorem of Erdös and Rado [3], we get a subset $F_{1}$ of $F_{0}$ of power $\eta$ consisting of pairwise compatible mappings. We may assume that the domains of mappings from $F_{1}$ are pairwise disjoint. For every $f \in F_{1}$, let

$$
\begin{aligned}
A_{f} & =\{\xi(\alpha, \beta), f(\xi(\alpha, \beta))=-1\}, \\
B_{f} & =\left\{\xi(\alpha, \beta), \alpha \in W, f(\alpha)=1, \beta<\kappa^{+}\right\} .
\end{aligned}
$$


It turns out that the power of every $A_{f}$ is at most $\kappa$ and that the order-type of every $B_{f}$ is at most $\kappa^{+} \cdot \kappa^{+}$. It follows from the regularity of $\Lambda^{+}=\eta$ that there is a sequence $f_{\alpha}, \alpha<\eta$, with the following property (writing $A_{\alpha}, B_{\alpha}$ in turn instead of $\left.A_{f_{\alpha}}, B_{f_{\alpha}}\right)$

$$
\left(\xi \in A_{\alpha} \& \xi \in A_{\beta}\right) \rightarrow \xi<\xi \text { holds for every } \alpha<\beta<\eta \text {. }
$$

As $\eta>\operatorname{sat}(C)$, we can construct a sequence $\eta_{\alpha}, \alpha<\eta$, satisfying (28). It follows from (33) that the order-type of $B_{\eta_{\gamma}}$, where $\gamma>\kappa^{+} \cdot \kappa^{+}$, is too small to have common elements with a sequence of $A_{\delta}$ 's of length $\gamma$. Hence $D$ satisfies the $\Lambda^{+}$-antichain condition.

According to Lemma 9, $D$ is the union of all $R(\alpha)$ 's and the rest of the proof of Theorem 5 is clear.

RemarKs. We have shown that every Boolean algebra can be embedded in a complete Boolean algebra with no rigid or homogeneous factors by an embedding preserving distributivity. It is natural to ask whether there are similar embeddings into rigid or into homogeneous Boolean algebras. It was shown in [13] that every complete Boolean algebra with a $\kappa$-closed dense subset can be completely embedded in a complete rigid Boolean algebra with a dense subset satisfying the same closure property. The results due to McAloon [7], Roguski [10] and others concerning the class of all hereditarily definable sets in Boolean valued models of set theory indicate that every $\kappa$-distributive Boolean algebra can be completely embedded in a $\kappa$ distributive rigid complete Boolean algebra but there is no algebraic proof of the fact. Koppelberg [5] proved that every Boolean algebra with a $\kappa$-closed dense subset can be completely embedded in a complete homogeneous Boolean algebra with a dense subset satisfying the same closure property. It remains an open problem whether every $\kappa$-distributive complete Boolean algebra can be completely embedded in a complete $\kappa$-distributive homogeneous Boolean algebra.

\section{REFERENCES}

1. W. B. Easton, Powers of regular cardinals, Ann. Math. Logic 1 (1970), 139-178.

2. R. Engelking and M. Karlowicz, Some theorems of set theory and their topological consequences, Fund. Math. 57 (1965), 275-285

3. P. Erdös and R. Rado, Intersection theorems for systems of sets, J. London Math. Soc. 35 (1960), $85-90$.

4. R. Jensen and $\mathrm{H}$. Johnsbraten, $A$ new construction of a nonconstructible $\Delta_{3}^{1}$ subset of $\omega$, Fund. Math. 61 (1974), 279-290.

5. S. Koppelberg, A lattice structure on the isomorphism types of complete Boolean algebras, Freie Universität Berlin, 1979, 41 pp., preprint.

6. S. Kripke, An extension of a theorem of Gaifman-Hales-Solovay, Fund. Math. 61 (1967), 29-32.

7. K. McAloon, Consistency results about ordinal definability, Ann. Math. Logic 2 (1971), 449-467.

8. R. McKenzie and J. D. Monk, On automorphism groups of Boolean algebras, Infinite and Finite Sets, Colloq. Math. Soc. János Bolysi, vol. 10, Keszthely (Hungary), 1973, pp. 951-988.

9. J. D. Monk and W. Rassbach, The number of rigid Boolean algebras, Algebra Universalis 9 (1979), 207-210.

10. S. Roguski, Extensions of models of ZFC to models of $Z F C+V=H O D$ with applications, Proc. Bierutowice Conf. (1975), Lecture Notes in Math., vol. 537, Springer-Verlag, Berlin and Heidelberg, 1976, pp. 241-248. 
11. M. Rubin, On the reconstruction of Boolean algebras from their automorphism groups, University of Colorado, Boulder 1978, 38 pp., preprint.

12. S. Shelah, Why there are many nonisomorphic models for unsuperstable theories, Proc. Internat. Congr. Math. (Vancouver 1974), Canadian Mathematical Congress, 1975, pp. 553-557.

13. P. Štěpanek, Cardinal collapsing and ordinal definability, J. Symbolic Logic 43 (1978), 635-642.

14. P. Štěpánek and B. Balcar, Embedding theorems for Boolean algebras and consistency results on ordinal definable sets, J. Symbolic Logic 42 (1977), 64-76.

Department of Cybernetics, Informatics and Operational Research, Charles University, Malostranské NÁmÉsti 25, 11800 Praha 1, Czechoslovakia 